diese als völlig steril erwiesen. Vielleicht sei der Gehalt an Albumosen und Peptonen von Einfluss auf diese Verflüssigung. Er erwähnt Versuche, im Innern der Kochkessel dieser Fabriken die Temperatur mit Thermoelementen festzustellen, wobei Temperaturen von $113^{\circ}$ beobachtet seien. Er fragt, woher es komme, dass manchmal einzelne Stücke einer Fleischkonserve eine rothe Farbe besässen; solches Fleisch sei minderwerthig im Geschmack.

Dr. Deichstetter.erklärt, dass jede Fleischkonserve beim Zersetzen eine Lachsfarbe annehme; durch nachheriges Sterilisiren werde sie wieder keimfrei.

Prof. Heyer hat in seinem Haushalt ebenfalls Rothfärbung des Fleisches beobachtet, namentlich dann, wenn dem Kochwasser zum Zwecke schnelleren Weichkochens Arak oder Kognak zugesetzt worden sei.

Dr. Möslinger hat ebenfalls öfters rothes Rindfleisch beobachtet, ohne eine Erklärung dafür zu finden; vielleicht sei die Art des Viehes die Ursache der Rothfärbung.

Der Vorsitzende kann diese Beobachtungen gleichfalls bestätigen und glaubt, dass die Rothfärbung mit dem verschiedenen Wassergehalt des Fleisches zusammenhänge.

Dr. Willemer erklärt, dass Fleischstücke, die unmittelbar auf Eis gelegen hätten, beim Kochen roth würden.

Dr. Deichstetter bestätigt letzteres.

Dr. von Liebig hat die Beobachtung gemacht, dass die Rothfärbung des Fleisches auf dem Lande sehr häufig, in der Stadt aber nie vorkomme, so dass jedenfalls in der Art der Aufbewahrung die Ursache dafür zu suchen sei.

Prof. Beckmann fragt, ob die von Dr. Wintgen beobachtete Verflüssigung der Gelatine direkt nach der Sterilisirung oder erst bei der Aufbewahrung der betr. Konserven aufgetreten sei; in letzterem Falle sei an die Wirkung chemischer Katalysatoren durch Gelatosenbildung zu denken.

Dr. Wintgen erklärt, dass die Verflüssigung direkt nach dem Sterilisiren beobachtet worden sei.

Dr. W indisch fragt, ob bei der Beobachtung der Rothfärbung der Salpetersäuregehalt des zum Kochen verwendeten Wassers berücksichtigt worden sei.

Nach einer Frühstïckspause von 11-12 Uhr folgte der Vortrag von Dr. Möslinger.

\title{
Ueber die Säuren des Weines und den Säurerïckgang.
}

$$
\text { Von }
$$

Dr. Möslinger in Neustadt a. d. Haardt.

Wenn ich, im Gegensatze zu sonstiger Gepflogenheit, mit dem Berichte über eine Arbeit vor diese Versammlung trete, noch ehe ich dieselbe für auch nur vorläufig abgeschlossen erklären kann, so geschieht es mit Rücksicht auf eine jüngst erfolgte Veröffentlichung von $R u d . K u n z^{1}$ ) „Ueber das Vorkommen und die Bestimmung der Milchsäure im Weine", worin theilweise der gleiche Gegenstand zur Behandlung gelangt ist.

Die Thatsache, dass Milchsäure in der grossen Mehrzahl aller auch der gesunden Weine in mehr oder minder erheblichen Mengen auftritt, ist in meinem

1) Diese Zeitschrift 1901, 4, 673 . 
Laboratorium seit längerer Zeit festgestellt und daselbst seit 1898, also seit jenem Zeitpunkte, wo wir uns ein brauchbares, weiter unten zu beschreibendes Verfahren der Milchsäurebestimmung ausgearbeitet hatten ${ }^{1}$ ), Gegenstand unausgesetzter und ausserordentlich oft wiederholter Beobachtung gewesen. Aber diese Thatsache, so bemerkenswerth sie an sich schon ist und so neu sie uns, hinsichtlich der gesunden und tadellosen Weine wenigstens, anfangs anmuthete, befriedigte uns nicht; viel wichtiger schien uns, die Ursache des Auftretens der Milchsäure und die Beziehungen derselben zu den übrigen Säuren des Weines $\mathrm{zu}$ verfolgen und womöglich klar zu legen.

Von den bekannten Säuren des Weines war für die Weinsäure durch die von $\mathrm{Halenke}$ und mir veröffentlichte und in der Praxis der Weinuntersuchung seither allenthalben bewäbrte Bestimmungsmethode die Möglichkeit sicherer analytischer Erfassung und Feststellung ihres Schicksales im Weine gegeben. Nicht so für die Aepfelsäure; in den Löslichkeitsverhältnissen ihrer Verbindungen analytisch verwerthbare Unterschiede gegen die anderen Säuren nicht darbietend, zwingt diese Säure den Analytiker zur Aufsuchung anders gearteter Unterscheidungsmerkmale. Es lag nahe, an die optischen Eigenschaften dieses Körpers zu denken. Aber in dieser Richtung liegende Versuche, die vor etwa zehn Jahren in Speyer meinerseits gemacht wurden und sich auf der Grundlage der Pasteur'schen Beobachtungen an äpfelsaueren Antimon-Ammonium bewegten, führten nicht zum Ziele. Erfolgreicher dagegen gestalteten sich Versuche, die ich in Gemeinschaft mit F. A u erbach alsbald nach Erscheinen der Walden'schen Arbeit ${ }^{2}$ ) über das polarimetrische Verhalten der aktiven Aepfelsäure unternahm. Wald en hat gezeigt, dass sich die an sich sehr schwache und schwankende Drehung der Aepfelsäure bei Gegenwart von Uran in alkalischer Lösung in eine konstante Linksdrehung von beträchtlicher Grösse, bis zum 450fachen Betrage der ursprünglichen Drehung, verwandeln lässt.

Ohne hier auf die technischen Einzelheiten des von uns angewendeten Verfahrens, deren Mittheilung und kritische Besprechung gelegentlich erfolgen wird, einzugehen, möchte ich nur einige der Beobachtungen mittheilen, die wir im Jahre 1897 an Weinen meiner Laboratoriumspraxis über deren Gehalt an aktiver Aepfelsäure machen konnten. Vergl. die Tabelle auf S. 1122.

Schon diese wenigen Beobachtungen, denen sich in der Folge zahlreiche weitere anschlossen, lehrten unzweifelhaft, dass die einzelnen Weine sehr verschiedene Mengen von aktiver Aepfelsäure enthalten, am meisten die sehr saueren Naturweine, die abgebauten weniger, bis auf Null herunter; ebenso ist in besseren, säurearmen Weinen, auch bei ausländischen Rothweinen, oft keinerlei aktive Aepfelsäure mehr vorhanden. In allen diesen Weinen musste nothwendig der Theil des "Säurerestes", der nicht Bernsteinsäure ist, von einer, von der gewöhnlichen Aepfelsäure abweichenden, inaktiven Säure gebildet sein. Die Frage

1) Vergl. meine Andeutung in dieser Zeitschrift 1899, 2, 106.

2) Ber. Deutsch. Chem. Ges. 1897, 30, 2889.

N. 01. 
nach der Natur dieser bisher nicht bekannten Säure blieb indessen zunächst eine offene.

Wir fanden in $100 \mathrm{ccm}$ :

Bezeichnung des Weines

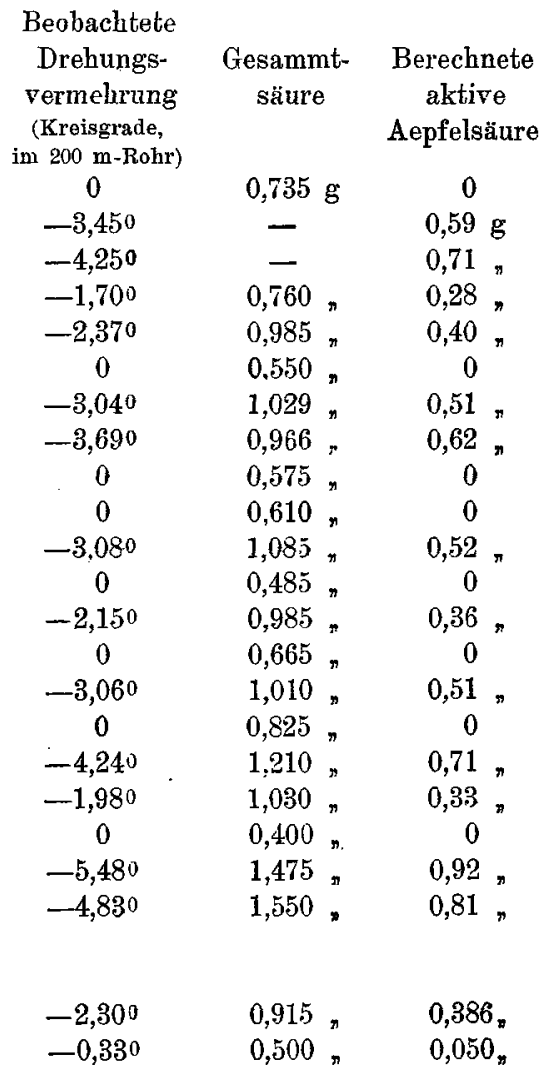

1896-er Edenkobener Naturwein

18.7-er Neustadter jíost

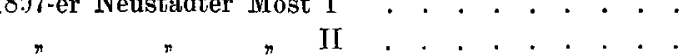

"Wein aus Most I . . . . . . . . . . . . .

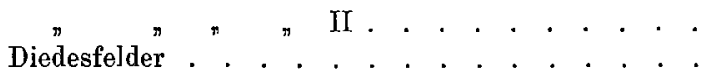

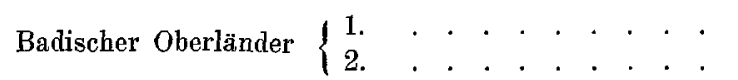

Italiener Rothwein .

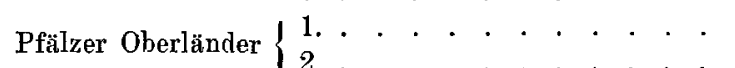

Mit Essigstich behafteter Wein . . . . . . . .

Friedelsheimer Riesling . . . . . . . . . . .

1893-er Bordeaux . . . . . . . . . . . . .

1896-er Freinsheimer Naturwein . . . . . . .

" Pfälzer Oberländer . . . . . . . . .

" Frankenwein . . . . . . . . . . . .

Elsässer . . . . . . . . . . . . .

1895-er Rheinhessischer Frankenwein $\left\{\begin{array}{l}\text { in Flaschen aufbewahrt . } \\ \text { aus dem Fass . . } \\ \text { mitZuckerlösung und Reinhefe } \\ \text { umgegohren und dabei im Ver- } \\ \text { hältniss } 500: 955 \text { verdünnt. }\end{array}\right.$ Aepfelwein

An diesem Punkte traf sich nun die Untersuchung mit den Ergebnissen einer anderen Beobachtungsreihe.

Die Nothwendigkeit, bei der Untersuchung der Weine und sogen. Kunstweine (Trester-, Hefen-, Rosinenweine u. s. w.) auf die Verwendung von Säurezusätzen Rücksicht zu nehmen, hatte mich dazu geführt, für den Nachweis der Citronensäure, Bernsteinsäure, Milchsäure leichter gangbare Wege, als die bis dahin bekannten, aufzusuchen.

Bezüglich der Citronensäure war mir dies gelungen ${ }^{1}$ ); bei der Milchsäure war, an einen qualitativen Nachweis aus verschiedenen Gründen nicht zu denken, hier musste sofort zur Aufsuchung einer brauchbaren quantitativen Trennung und Bestimmung geschritten werden. Und zwar war hierbei von einer Aetherextraktion der Eindampfrückstände des Weines womöglich abzusehen; gerade an der Unhandlichkeit und Unsicherheit hierauf begrïndeter Verfahren

1) Diese Zeitschrift $1899,2,105$. 
waren ja die bisherigen Milchsäurebestimmungen im Weine im Wesentlichen gescheitert.

In der leichten Löslichkeit des Baryumlaktates in selbst starkem Alkohol (von 70-80 Vol.-0\%) fanden wir bald das geeignete Mittel zur Trennung der Milchsäure von den übrigen nicht flüchtigen Säuren des Weines, deren Baryumsalze in Alkohol dieser Stärke vollkommen unlöslich sind. Die Schwierigkeit, dass bei Absättigung der Säuren des Weines mittelst Barytwasser nicht bloss die Baryumsalze, sondern wegen des Vorhandenseins organisch sauerer Alkalien (Weinstein, Kaliummalat u. s. w.) auch die neutralen Alkalisalze eben dieser Säuren entstehen, war entweder dadurch zu beheben, dass man den Wein auf geeignete Weise von seinen Mineralbestandtheilen befreite, oder dadurch, dass man vor der Trennung der Barytsalze durch Umsetzung mit überschüssigem Chlorbaryum die vollständige Ueberführung der Säuren in ihre Barytsalze erzwang. Wir wählten anfänglich diesen letzteren Weg, haben aber in der Folge dem Verfahren der Entfernung der Mineralbestandtheile unter gewissen Umständen den Vorzug gegeben, nämlich immer dann, wenn es sich darum handelt, sehr geringe Mengen ron Milchsäure oder letztere in Gegenwart grösserer Mengen Zucker (in Most oder jungem Wein u. dergl.) zu bestimmen.

Das Verfahren der Milchsäure-Bestimmung gestaltete sich bei Zuhilfenahme von Chlorbaryum behufs Umsetzung in die Barytsalze wie folgt:

Aus 50 oder $100 \mathrm{ccm}$ des $\mathrm{zu}$ untersuchenden Weines wird in bekannter Weise mittelst Wasserdampf die flüchtige Säure abgeblasen und die zurückbleibende Flüssigkeit in einer Porcellanschale mit Barytwasser bis zur neutralen Reaktion gegen Lackmus abgesättigt. Nach dem Hinzufügen von $5-10 \mathrm{ccm}$ $10 \%$-iger Chlorbaryumlösung wird bis auf etwa $25 \mathrm{ccm}$ eingedampft und mit einigen Tröpfchen Barytwasser aufs Neue genaue Neutralität hergestellt. Man fügt nun vorsichtig in geringen Mengen unter Umrühren reinsten $95 \%$-igen Alkohol hinzu, bis die Flüssigkeit etwa $70-80 \mathrm{ccm}$ beträgt, und führt den Inhalt der Porcellanschale nunmehr unter Nachspülen mit Alkohol in einen $100 \mathrm{ccm}-$ Kolben über, füllt mit Alkohol auf und filtrirt durch ein trockenes Faltenfilter, wobei der Trichter bedeckt gehalten wird. $80 \mathrm{ccm}$ oder mehr des Filtrates werden unter Zusatz von etwas Wasser in einer Platinschale verdampft, der Rückstand wird alsdann vorsichtig verkohlt und - ohne die Asche weiss zu brennen, was überflüssig ist - seine Alkalität mit 1/2-N.-Salzsäure in bekannter Weise bestimmt und in ccm N.-Alkalilauge ausgedrückt. $1 \mathrm{ccm}$ Aschen-Alkalität entspricht $0,090 \mathrm{~g}$ Milchsäure oder, wenn diese in Weinsäure umzurechnen ist, $0,075 \mathrm{~g}$ Weinsäure.

Zieht man es vor, die Mineralstoffe $\mathrm{zu}$ beseitigen, ehe man an die Ueberführung in die Baryumsalze und deren Trennung geht, so wird wiederum aus 50 oder $100 \mathrm{ccm}$ Wein die flüchtige Säure abgetrieben, der Rückstand in der Schale mit etwas Weinsäure versetzt, wozu meist 0,2 bezw. $0,4 \mathrm{~g}$ ausreichen, und bis zum dünnen Syrup (d. h. bis auf wenige ccm) eingedampft. Man giesst den Rückstand in einen mit Glasstopfen versehenen $50 \mathrm{ccm}$ fassenden graduirten 
Stehcylinder, spült mit wenigen Tropfen Wasser bis das Volumen der wässerigen Flüssigkeit etwa $5 \mathrm{ccm}$ beträgt, und darauf weiter mit kleinen Mengen von 95\%-igem Alkohol nach, immer unter Umschütteln, bis die Flüssigkeit $30 \mathrm{ccm}$ beträgt; alsdann fügt man zweimal je $10 \mathrm{ccm}$ Aether hinzu, indem man jedesmal kräftig schüttelt. Das Gesammtvolumen beträgt nunmehr $50 \mathrm{ccm}$. Man schüttelt und lässt absitzen, bis die Flüssigkeit völlig klar geworden, giesst in eine Porcellanschale ab und spült mit Aether-Alkohol nach. Unter Zusatz von Wasser wird die Flüssigkeit nunmehr zunächst von Aether und Alkohol durch Eindampfen befreit, alsdann mit Barytwasser neutralisirt und - ohne Zusatz von Chlorbaryum - weiter verfahren wie oben.

Die theoretische Grundlage dieser Milchsäurebestimmung bei Gegenwart der anderen bekannten Säuren des Weines erscheint zunächst völlig einwandfrei. Der oft wiederholte blinde Versuch, angestellt an künstlichen Gemischen der Säuren und Salze des Weines, ergab thatsächlich 0 ; bei Zusatz bekannter Mengen von Milchsäure wurden etwa 98\% der angewendeten Mengen wiedererhalten. Nichtsdestoweniger muss ich ausdrücklich bemerken, dass bei der Anwendung des Verfahrens auf den Wein selbst sich bis auf Weiteres gewisse Fehlerquellen nicht vermeiden lassen, als deren Wirkung es anzusehen ist, dass man nicht mehr als etwa einige $90 \%$, bestenfalls $95 \%$ der vorhandenen Milchsäure durch das Verfahren zu erbalten vermag. Wir haben diese Verhältnisse während mehrerer Jahre kritisch untersucht und dabei sehr beachtenswerthe Thatsachen kennen gelernt; es würde indessen für die Zwecke dieser Mittheilung viel zu weit führen, wollte ich hierauf des Näheren eingehen. Dennoch möchte ich wenigstens die hauptsächlichsten Fehlerquellen andeuten, welche der analytischen Genauigkeit entgegenstehen. Die Fehlerquellen liegen einmal in dem Umstande, dass jedes Erhitzen und Findampfen des Weines im saueren $\mathrm{Zu}$ stande eine mehr oder weniger weitgreifende, immer aber merkliche innere Veresterung vorhandener Milchsäure zur Folge hat und dass die Baryumverbindungen dieser ebenfalls saueren Veresterungsprodukte von dem zur Trennung dienenden Alkohol theilweise mit niedergerissen werden. Die Rückbildung der Milchsäure aus diesen Körpern durch Erhitzen mit Alkali herbeiführen zu wollen, geht deshalb nicht an, weil selbst geringe Mengen vorhandenen Zuckers unter der Wirkung des Alkalis schon sehr merkliche Mengen Milchsäure abspalten. Eine weitere Fehlerquelle eröffnet sich durch das Vorhandensein grösserer oder geringerer Mengen von Eiweissbasen und unter Umständen ron Ammoniak im Wein; derjerige Antheil der Milchsäure, welcher bei der Absättigung des Weines mit Barytwasser mit diesen Körpern in Verbindung tritt und so in den Trennungsalkohol iibergeht, entzieht sich der Bestimmung durch das beschriebene Verfahren. Dass endlich durch nicht sorgsame Ausführung der Verkohlung gleichfalls Fehler entstehen können, ist leicht verständlich. Mit der letztgenannten Fehlerquelle ist natürlich diejenige Abänderung des Verfahrens, bei der die Mineralstoffe weggeschafft werden, nicht behaftet.

Obgleich nicht frei von Fehlern, wie man sieht, und den Ansprüchen an 
höchste analytische Genauigkeit nicht genügend, besitzt diese Milchsäure-Bestimmung doch den grossen Vorzug schneller Ausführbarkeit und grosser Einfachheit gegenüber den bisherigen, übrigens noch weitaus ungenaueren Verfahren. Sie hat sich denn auch in der Folge vorzüglich bewährt und uns in den Stand gesetzt, ausserordentlich wichtige Beobachtungen zu machen.

Die Absicht, auf diesem Wege Zusätze von Milchsäure zum Weine nachzuweisen, hat allerdings sofort wieder aufgegeben werden müssen, nachdem sich alsbald bis zur Gewissheit herausgestellt hatte, dass milchsäurefreie Weine nur selten und in einem ganz bestimmten Stadium des Weines angetroffen werden. Was wir auch untersuchten, welcher Herkunft und welcher Qualität auch immer die untersuchten Weine waren, überall begegneten wir in geringeren oder grösseren Mengen der Milchsäure, die im Laufe unserer Untersuchungen mehr und mehr zum normalen Weinbestandtheil heraufrückte, im Gegensatze zu der bis dahin herrschenden Anschauung, dass sie ihre Gegenwart stets unregelmässigen Gährungsvorgängen verdankt und mit ihrem Auftreten in-irgend erheblicherer Menge den Wein zu einem kranken stempelt.

Nur um ein Beispiel zu geben, in welchen Mengen man der Milchsäure auch in gänzlich normalen Weinen begegnet, hebe ich eine kleine Anzahl unserer Milchsäure-Bestimmungen aus den Jahren 1897 und 1898 heraus. Die Weine enthielten $\mathrm{g}$ in $100 \mathrm{ccm}$ :

\begin{tabular}{|c|c|c|c|c|c|c|c|}
\hline No. & $\begin{array}{c}\text { Bezeichnung } \\
\text { des } \\
\text { Weines }\end{array}$ & $\begin{array}{c}\text { Freie } \\
\text { Säure } \\
\text { (Gesammt- } \\
\text { säure) }\end{array}$ & $\begin{array}{l}\text { Milch- } \\
\text { säure }\end{array}$ & No. & $\begin{array}{c}\text { Bezeichnung } \\
\text { des } \\
\text { Weines }\end{array}$ & $\begin{array}{c}\text { Freie } \\
\text { Säure } \\
\text { (Gesammt. } \\
\text { sãure) }\end{array}$ & $\begin{array}{l}\text { Milch- } \\
\text { säure }\end{array}$ \\
\hline 75. & Rothwein: Portugieser & 0,535 & 0,216 & - & Pfälzer Naturwein & 0,75 & 0 \\
\hline 00. & $n$ & 0,5 & 0,1 & - & , & 0,70 & 126 \\
\hline 597. & Weisswein: Dürkhe & 0,6 & & - & $n$ & 0,0 & 0,280 \\
\hline 一 & Köni & 0,4 & & - & Bocksteiner (Flasche & & 0,29 \\
\hline $69 a$. & Gezuckerte Oberländer & 0, & & - & Bordeaux & 0 , & 0,30 \\
\hline 624. & n & 0,7 & 5 & - & Clairet (Lothringer) & 0,95 & 0,22 \\
\hline $19 a$. & , & 0,395 & 0,146 & 1058. & Deidesheimer Naturwein & - & 0,221 \\
\hline - & " & 0,500 & 0,234 & 1062. & . & - & 0,244 \\
\hline
\end{tabular}

Diese Befunde wiederholten sich in der Folge in ungezählten Fällen. Nur lernten wir auch hin und wieder Weine kennen, die frei waren von Milchsäure oder nur Spuren aufwiesen. Einige davon seien gleichfalls hier aufgeführt. Es enthielten $\mathrm{g}$ in $100 \mathrm{ccm}$ :

\begin{tabular}{|c|c|c|c|c|c|c|c|c|}
\hline & $\begin{array}{c}\text { Bezeichnung } \\
\text { des } \\
\text { Weines }\end{array}$ & $\begin{array}{c}\text { Freie } \\
\text { Säure } \\
\text { (Gesammt- }\end{array}$ & $\begin{array}{l}\text { Milch- } \\
\text { sãure }\end{array}$ & & $\begin{array}{l}\text { zeichnung } \\
\text { des } \\
\text { Hostes }\end{array}$ & & $\begin{array}{c}\text { Freie } \\
\text { Sänre } \\
\text { Gesammt- }\end{array}$ & $\begin{array}{l}\text { Mileh- } \\
\text { săurur }\end{array}$ \\
\hline \multirow{5}{*}{$\begin{array}{l}\text { Jung- } \\
\text { wein }\end{array}$} & Thüngertsheime & 1,55 & 0,048 & \multirow{5}{*}{$\begin{array}{c}\text { Frischer } \\
\text { Most }\end{array}$} & (1898-er) & Gutedel & 1,075 & 0 \\
\hline & Deidesheimer & 0,96 & 0 & & (1899-er) & Neinstadter & & \\
\hline & & 0,99 & 0 & & (Oesterr & r. u. Riesling & g) 0,900 & 0 \\
\hline & Gleisweilerer & 1,05 & 0 & & Malingre & & 1,200 & 0 \\
\hline & $\pi$ & 1,10 & 0,050 & & (1900-er) & Portugieser & r 1,105 & 0,01 \\
\hline
\end{tabular}

Von den weiteren Untersuchungen seien nur noch die höchsten Befunde erwähnt: Als böchster Milchsäuregehalt in noch normalem Wein wurde $0,60 \mathrm{~g}$ 
in $100 \mathrm{ccm}$ festgestellt; bei anderen Weinen wurden darüber hinaus sogar Mengen von $0,9-1,0 \mathrm{~g}$ beobachtet, indessen mussten diese letzteren Weine nach ihrem ganzen äusseren Verhalten und ihrer Lebensgeschichte als „milchsäurestichig" bezeichnet werden.

Wenn nach unseren Erfahrungen sehr bald als feststehend angenommen werden konnte, dass die Milchsäure als wesentlicher Bestandtheil aller möglichen Weine anzusehen war, da man ihr in gleicher Weise in edlen und geringwerthigen Weinen der rerschiedensten Herkunft, in Rothweinen und Weissweinen, in süssen und trockenen, in natürlichen und gezuckerten, in Rosinen-, Trester-, Hefeweinen, sowie Obstweinen, und zwar manchmal in erstaunlicher Menge begegnete, so traten andererseits alsbald auch zwischen der Milchsäure and den iibrigen Verbältnissen der Weine gewisse innere Beziehungen hervor, die zur Annabme eines ursächlichen Zusammenhanges berechtigten.

Insbesondere musste auffallen, dass in den älteren Weinen, trotz vollständig normaler Beschaffenheit, die Milchsäure in immer grösserer Menge sich zeigte; dass im Einklange damit die Moste gar keine, die Jungweine z. Th. ebenfalls keine oder nur geringe Mengen aufwiesen, dass man ihr immer dann in auffallenden Mengen begegnete, wenn ein starker Abbau des Weines verbunden mit erheblichem Säurerückgang stattgefunden hatte und gleichzeitig der Gehalt an aktiver Aepfelsäure ganz oder grösstentheils verschwunden war.

Zweifellos stand vor Allem fest, dass die in solcher Allgemeinheit den Weinen innewohnende Milchsäure nicht etwa der bekannten Thätigkeit solcher Organismen zuzuschreiben war, die aus vorhandenem Zucker Milchsäure bilden, obgleich wir gerade in dieser Richtung sehr bemerkenswerthe und mit den herrschenden Anschauungen durchaus nicht übereinstimmende Erfahrungen betreffend den Uebergang von Zucker in Milchsäure hatten machen können, unter Umständen, welche eine solche Bildung auszuschliessen schienen. Vorgänge ähnlicher Art aber waren bei einer grossen Zahl der untersuchten, milchsäurehaltigen Weine geradezu ausgeschlossen; bei ihnen hatte ein völlig normaler Verlauf der Gährung einen gesunden, so .gut wie zuckerfreien und auch später durch Krankheitserscheinungen ’oder Fehler in keiner Weise gestörten Wein ergeben. Hier lag eine andere Annahme nahe: Diese Milchsäure war nicht durch und unter Zuwachs von Säure in den Wein gerathen, sondern sie musste in einer Umbildung bereits vorhandener Säure ihre Entstehungsursache haben. Und dass von den Säuren des Weines hier in erster Linie die Aepfelsäure in Betracht kommt, ist selbstverständlich; sie ist die einzigè, deren Menge gross genug ist, um eine solche Bildung zu ermöglichen, sie ist auch diejenige, welche vom Säureriickgang beim Abbau der Weine am meisten betroffen wird. Dass auch der atomistische Bau der Aepfelsäure eine solche Annahme mindestens nicht ausschliesst, insofern ein Molekül derselben gerade die Bestandtheile je eines Moleküles Milchsäure und Kohlensäure enthält, sei nur beiläufig erwähnt; ich bin nämlich noch nicht sicher, dass der Vorgang in 
der Wirklichkeit immer die so einfache Gestalt des blossen Zerfalls annimmt, wie sie durch die nachstehende Gleichung wiedergegeben wird:

$$
\mathrm{C}_{4} \mathrm{H}_{6} \mathrm{O}_{5}=\mathrm{CO}_{2}+\mathrm{C}_{3} \mathrm{H}_{6} \mathrm{O}_{3} \text {. }
$$

Wenn diese ganze Anschauung überhaupt Berechtigung haben sollte, so musste vor Allem ein klarer Zusammenhang zwischen Säurerückgang und Auftreten von Milchsäure beobachtet werden können.

In dieser Hinsicht nun hat die unausgesetzte Beobachtung eines von mir selbst im Herbste 1899 als Most in vier Halbstücken eingekellerten und natürlich belassenen Gleisweilerer Weines jeden bis dahin möglichen $Z_{\text {weifel beseitigt. }}$

Dieser Wein, welcher von durchaus gesunden Trauben stammte und als Jungwein 11,3\% Säure aufwies (nach dem Ausfallen des Weinsteines; - vorher zeigte er etwa $13 \%$ Säure), besass, wohl in Folge der ihm angediehenen sorgfältigen Kellerbehandlung, anfangs die hier unwillkommene Eigenschaft, seine Säure mit grosser Zähigkeit beizubehalten; er war zu den Vorgängen des Abbaues offenbar nur wenig geneigt. Sein mehrfach bestimmter Gehalt an Michsäure war dabei so spurenhaft niedrig, dass man ihn gleich Null setzen konnte; dieser Zustand währte den ganzen Winter $1899 / 1900$ hindurch. Erst nach dem dritten Abstich begannen in einzelnen Fässern sich Anfänge des Säurerückganges zu zeigen; im Juli 1900 begann die Säure auf $11,00^{\circ}{ }^{\prime} 00$ zu sinken, um dann im Laufe des Herbstes und darauffolgenden Winters und Sommers allmählich auf $8,1 \%$ herabzugehen. Zugleich aber wurde Milchsäure in immer steigenden und dem Säurerückgang durchaus parallel gehenden Mengen nachgewiesen; es wurde nämlich gefunden:

Zeit der Untersuchung

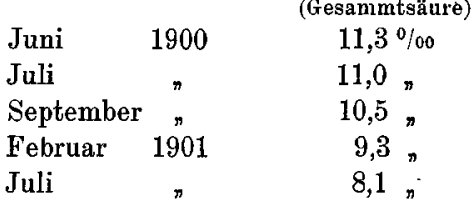

Milchsäure

(berechnet als Weinsäure)

$$
0
$$

$0,40 \%$

0,84,

1,95

2,75 ,
Säurerückgang

0
$0,3^{\circ}: 00$
$0,8 \%$
$2,0 \%$
$3,2 \%$

Dabei ist zu bemerken, dass der Gehalt des Weines an Gesammtweinsteinsäure wesentlich der gleiche geblieben war; weder in seiner äusseren Erscheinung noch in seinen sonstigen Eigenschaften bot der Wein Anzeichen für die mit ihm vorgegangene innere Veränderung.

Gerade in diesem Falle erschien es uns übrigens von Bedeutung, den Nachweis zu führen, dass man es hier thatsächlich mit nichts Anderem, denn mit Milchsäure zu thun hatte. Zu diesem Zwecke wurden $200 \mathrm{ccm}$ dieses Weines nach dem Vertreiben der Essigsäure mit Barytwasser neutralisirt, auf ein Viertel eingedampft, mit Schwefelsäure in schwachem Ueberschuss versetzt und im Scheidetrichter dreimal mit grösseren Mengen Aether ausgeschüttelt.

Nach dem Verdunsten des Aethers wurde die wässerige Lösung des Rückstandes noch einmal der gleichen Behandlung (mehrfacher Ausschüttelung mit Aether) unterworfen, das Gemisch der schliesslich wieder in wässerige Lösung übergeführten Säuren mit überschüssigem Barytwasser erhitzt und nach wieder 
bewirkter Neutralisation durch verdünnte Schwefelsäure die Trennung der Baryumsalze durch Alkohol in der oben beschriebenen Weise vorgenommen. Das Gewicht des aus der alkoholischen Lösung durch Trocknen bei $100^{\circ} \mathrm{C}$. bis zum gleichbleibenden Gewicht erhaltenen Rückstandes betrug 0,4788 g. Hieraus wurden durch Veraschen gewonnen 0,298 g Baryumkarbonat oder 62,20\% vom Gewichte des Baryumsalzes. Die Theorie erfordert für Baryumlaktat $62,54 \%$; es herrscht also befriedigende Uebereinstimmung. - Bei diesem Vorgehen war nicht ganz die Hälfte der vorhandenen Milchsäure gewonnen worden.

Im Lichte dieser wichtigen und nunmehr sicher gestellten Erfahrung, dass mit dem Säurerückgang Schritt um Schritt Auftreten und Vermehren von Milchsäure Hand in Hand geht, und zwar in Mengen, welche der gehegten Vermuthung durchaus entsprachen, gewinnen die übrigen, an Weinen aller Art ermittelten Milchsäurebefunde erhöhte Bedeutung und zugleich eine interessante Erklärung. Wir lernen nunmehr verstehen, warum wir der Milchsäure allenthalben in den Weinen, nicht bloss in den fehlerhaften, begegnen müssen; wir begreifen, dass diese Säure besonders kennzeichnend ist für stark abgebaute Weine, dass sie in Mosten stets, in jungen Weinen nicht selten fehlt, und wir müssen zugestehen, dass ihr Auftreten im Weine des bisherigen Charakters der Ungehörigkeit vollkommen entkleidet ist.

Es würde mich für heute zu weit führen, wollte ich auf alle die vielfachen Versuchsreihen, welche zur weiteren Aufklärung über die Bedeutung der Milchsäure im Weine unsererseits angestellt wurden, hier eingehen. Auch sind wir noch mitten in der Arbeit; die bereits erkannte wichtige Rolle der Temperatur und des Mehr oder Weniger an Alkohol bei dem hier besprochenen Vorgange der Milchsäurebildung harrt noch der genaueren Feststellung. Ebenso bedarf die Thatsache, dass aus grau- und sauerfaulen Trauben gewonnene Weine auch in ganz jungem Zustande bereits erhebliche Mengen von Milchsäure aufweisen können und dass manche Weine einem auffallend raschen Säurerückgang schon während oder kurz nach der Hauptgährung unter Milchsäurebildung unterliegen, der genaueren Verfolgung. Auch bleibt die Frage, ob neben der aktiven Aepfelsäure von vornherein oder als Begleiterscheinung ihrer Umbildung in Milchsäure eine inaktive Form der Aepfelsäure einhergeht oder entsteht, noch immer offen, genau so offen, wie die Frage nach der näheren Ursache des Zerfalles der Aepfelsäure überhaupt.

Immerhin ist eine Reihe von Thatsachen festgelegt und ich möchte die aus meinen bisherigen Beobachtungen zu ziehenden Schlüsse in folgende Sätze zusammenfassen :

1. Die Milchsäure ist ein zwar nicht stets, aber doch sehr allgemein in den Weinen verschiedenster Herkunft und Herstellung auftretender, der Menge nach oft sehr wesentlicher Bestandtheil.

2. Die Ursachen für das Auftreten der Milchsäure im Wein lassen sich in zwei Gruppen scheiden. Die Milchsäure des Weines entsteht: 
a) Durch die specifische „Milchsäuregährung“, verursacht durch die bekannten Organismen dieser Gährung.

Hierbei wird vorhandener Zucker in Milchsäure umgesetzt, deshalb erfahren zuckerfreies Extrakt und Säuregehalt eine Zunahme. Diese Milchsäuregährung kann in geringem, zuweilen auch erheblicherem Maasse neben der alkobolisehen Gährung einhergehen.

Hierher gehören unter Anderem alle die Fälle, in denen Milchsäure als Folge unreiner Gährung des Weines, besonders z. B. bei der Vergährung auf den Hülsen, bei beginnendem "Stich" in der Hauptgährung, bei zu starkem Erwärmen während der Gährung u. s. w., kurz in Folge der sog. Gährfehler, schon im ganz jungen Weine trotz gesunder Trauben zu beobachten ist.

b) Beim Ab. oder Ausbau des Weines, namentlich des alkoholarmen Weines, aus dem Zerfall oder sonstiger Umbildung der Aepfelsäure.

Kennzeichnend für diesen Vorgang ist die Abnahme des zuckerfreien Extraktes um mindestens den halben, und die $\mathrm{Ab}$ nahme der freien Säure des Weines um mindestens den ganzen Betrag der auftretenden Milchsäure (freie Säure und Milchsäure in Weinsäure ausgedrückt).

Dieser Art ist die Herkunft aller der Milchsäure, die in gesunden, fehlerfreien, aus normaler Gährung und gesunden 'Trauben hervorgegangenen Weinen gefunden wird.

3. Traubenmost, auch derjenige von grau- und sauerfaulen Trauben, enthält keine merklichen Mengen von Milchsäure.

4. Die normale und reine alkoholische Gährung, wie sie z. B. durch Reinzuchthefe in sterilisirten Mosten oder sterilisirten Gemischen von Wein und Zuckerlösung bewirkt wird, lässt keine merklichen Mengen von Milchsäure entstehen.

Im Gegensatze hierzu sieht man, wie unter 2 a erwähnt, bei der spontanen Gährung der Moste und Weine in der Praxis, je nach dem zufälligen Grade der Reinheit dieser Gährung meist geringe, manchmal auch erheblichere (bis zu 0,4\% ansteigende) Mengen von Milchsäure auftreten.

Wie ich weiter oben bemerkte, harren im Anschluss an den Milchsäurebefund im Weine wichtige andere Fragen ihrer Bearbeitung und Lösung. Aber eines ist jetzt schon gewiss, bei Beurtheilung der Weine auf Grund der Analyse wird künftighin auch die Bestimmung der Milchsäure nicht selten beranzuziehen sein und hierzu das angegebene Verfahren eine bequeme und bei nicht allzuhohen Ansprüchen genügende Handhabe bieten.

Auf alle Fälle aber ist mit dem Nachweise des eigenartigen Zusammenhanges zwischen Säurerückgang und neugebildeter Milchsäure ein nicht unwesentlicher Theil jenes Dunkels gelichtet, das bisher über dem sog. Säurerückgang, dem scheinbar spurlosen Verschwinden eines Theiles der Säure des Weines, geschwebt hat. 
Ich möchte anch für heute nicht schliessen, ohne vorher noch den Kollegen Dr. Auerbach, Dr. Eckstein und Dr. Werner, die mich bei Beginn und Fortsetzung dieser Arbeiten aufs Wirksamste unterstützten, meinen Dank auszusprechen.

$$
\text { Diskussion. }
$$

Prof. Beckmann fragt an, ob thatsächlich Hydracrylsäure entstehe.

Dr. Möslinger erklärt, dass die Unterscheidung der Milchsäuren sehr schwer sei und stellt diesbezügliche Versuche in Aussicht.

\title{
Ueber Gelee-Extrakt.
}

\author{
Von
}

\section{A. Bömer in Münster i. W.}

Neuerdings kommen unter der Bezeichnung ,Ge lee-Extrakt zur schnellen

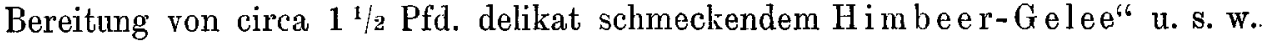
Präparate in kleinen Kartons in den Handel, auf deren Rückseite folgende Gebraucbsanweisung beigefügt ist :

${ }_{n}^{1 / 2} 1$ Wasser wird mit 150-200 g ungeblautem Zucker und den in diesem Päckchen. vorhandenen beiden Pulvern gut aufgekocht; ist dieses geschehen, so nimmt man das. Gekochte vom Feuer, thut den Inhalt des kleinen Fläschchens hinein, rührt es nochmals. gut durch und schüttet das nun fertige Frucht-Gelee, nachdem es etwas abgekühlt, in eine hübsche Glasform oder Krystallschale und lässt es erstarren."

In jedem Karton befinden sich zwei Pulver und ein kleines Fläschchen mit einer Flüssigkeit. Das grössere Pulver besteht aus Gelatine, welche mit einem je nach der Art der ,Frucht" verschiedenen Farbstoffe versehen ist, das zweite, kleinere Pulver dagegen aus Citronensäure oder Weinsäure. Der Inhalt des kleinen Fläschchens endlich ist ein je nach der bezeichneten Frucht verschiedener künstli cher Fruchtäther.

Es fragt sich nun, wie diese Gelee-Extrakte in Bezug auf den $\$ 10$ des Nahrungsmittel-Gesetzes zu beurtheilen sind.

Es handelt sich meines Erachtens bei den Gelee-Extrakten z. B. bei dem Gelee-Extrakt zur Bereitung von $\mathrm{Himbeer-Gelee} \mathrm{nicht} \mathrm{um} \mathrm{den} \mathrm{Himbeer-}$ Gelee im gewöhnlichen Sinne des Wortes, d. h. um Himbeer-Gelee, wie er in der Regel durch Einkochen von Himbeersaft mit viel Zucker gewonnen wird, und der in Folge seines natürlichen Gehaltes an gelatinirenden Stoffen (Pektinstoffen) nach dem Erkalten zu einem Gelee, dem Himbeer-Gelee, erstarrt. Bei dem hier in Frage stehenden Himbeer-Gelee handelt es sich meines Erachtens. um Hịbeer-Gelee oder Himbeer-Gallerte - auch wohl süsse klare Sulze genannt -, wie sie im Haushalte aus Himbeersaft unter Zusatz gelatinirender Mittel (Gelatine, Agar-Agar) hergestellt und als sog. süsse Speise zum Nachtisch mit einer passenden Sauce genossen werden. - Vergl. Da vidis Kochbuch 37. Aufl. 1899, S. 391. 